\title{
Schistosoma mansoni major egg antigen Smp40: molecular modeling and potential immunoreactivity for anti-pathology vaccine development
}

\author{
Mohamed F Abouel-Nour/ ${ }^{+}$, Mahmoud Lotfy*, Abdelfattah M Attallah**, \\ Barbara L D oughty***
}

\begin{abstract}
Zoology Department, Faculty of Science, Mansoura University, Mansoura, Egypt *Molecular and Cellular Biology Department, Genetic Engineering and Biotechnology Research Institute, Minufiya University, Sadat City, Minufiya, Egypt

**Research and Development Department, Biotechnology Research Center, New Damietta, Egypt ***Pathology Research, The University of Texas Medical Branch at Galveston, Galveston, TX, US
\end{abstract}

The pathogenesis of Schistosoma mansoni infection is largely determined by host T-cell mediated immune responses such as the granulomatous response to tissue deposited eggs and subsequent fibrosis. The major egg antigens have a valuable role in desensitizing the $C D 4^{+}$Th cells that mediate granuloma formation, which may prevent or ameliorate clinical signs of schistosomiasis. S. mansoni major egg antigen Smp40 was expressed and completely purified. It was found that the expressed Smp40 reacts specifically with anti-Smp40 monoclonal antibody in Western blotting. Three-dimensional structure was elucidated based on the similarity of Smp40 with the small heat shock protein coded in the protein database as ISHS as a template in the molecular modeling. It was figured out that the C-terminal of the Smp40 protein (residues 130 onward) contains two alpha crystallin domains. The fold consists of eight beta strands sandwiched in two sheets forming Greek key. The purified Smp40 was used for in vitro stimulation of peripheral blood mononuclear cells from patients infected with $\mathrm{S}$. mansoni using phytohemagglutinin mitogen as a positive control. The obtained results showed that there is no statistical difference in interferon- $\gamma$, interleukin (IL)-4 and IL-13 levels obtained with Smp40 stimulation compared with the control group $(\mathrm{P}>0.05$ for each). On the other hand, there were significant differences after Smp40 stimulation in IL-5 (P = 0.006) and IL-10 levels ( $\mathrm{P}<0.001)$ compared with the control group. Gaining the knowledge by reviewing the literature, it was found that the overall pattern of cytokine profile obtained with Smp40 stimulation is reported to be associated with reduced collagen deposition, decreased fibrosis, and granuloma formation inhibition. This may reflect its future prospect as a leading anti-pathology schistosomal vaccine candidate.

Key words: Schistosoma mansoni- soluble egg antigen - cytokines - Smp40 - molecular model

Schistosomiasis is a debilitating parasitic disease affecting about 200 million people in 70 countries in the world and is caused by one of the three different species of Schistosoma: S. mansoni, S. haematobium, and S. japonicum. The major pathology of these parasitic infections is associated with a host delayed type hypersensitivity reaction to parasitic egg and egg products. Granulomatous inflammation is a cellular hypersensitivity reaction mediated by egg antigen-specific, MHC class II-restricted, TCR $\alpha \beta$ expressing, CD4 ${ }^{+}$T helper cells (Iacomini et al. 1995). Patients infected with $S$. mansoni mount cellular and humoral immune responses to soluble egg antigens derived from crude homogenates of eggs. Thus, the end result of host responses to schistosome eggs in the liver is advanced portal fibrosis with dense deposits of collagens in greatly expanded portal tracts (El-Zayadi 2004). The immune reaction produced by the body against the schistosomal infection is a double-weaponed arm. Unfortunately, the harmful weapon is the longest and the

+Corresponding author: abouelnour@hotmail.com

Received 1 November 2005

Accepted 10 May 2006 most powerful. That is the immune reaction against the schistosomal egg causing the schistosomal granuloma. The other weapon of the immune system that should be lengthened and empowered is the protective immune response against infection, egg production and/or the granuloma formation. Many researchers have been doing their best to get the suitable agent that can stimulate the maximal, specific immune response against schistosomiasis (Goes \& Hirsch 1996).

Considerable efforts have been exerted to determine which $S$. mansoni antigens induce and elicit T cell-mediated responses and granuloma formation (Goes \& Hirsch 1996). Several laboratories have isolated various antigens from crude soluble egg anigens (SEA) and soluble worm antigens (SWAP), and investigated their role in serology, blastogenic reactions, and granuloma responses to SEA (Bahia-Oliveira et al. 1997). These studies revealed a variety of biologically active antigenic moeities derived from S. mansoni antigen preparations (Goes \& Hirsch 1996). One antigen, Smp40 (major egg antigen p40), has been described as highly immunogenic in humans and has been cloned and sequenced (Cao et al. 1993). The Smp40 peptide has 354 amino acid residues and shares homologies with the family of heat shock proteins and $\alpha$-crystallins. There is evidence that $\alpha$-crystallins act as chaperone for other important egg antigens released during the migra- 
tion phase of the eggs in the hepatic system (Nene et al. 1986). The immune response to Smp 40 and Smp 40 overlapping peptides can be studied in the cellular proliferation assays with the addition of either anti-interleukin (IL)10 or IL-2 to overcome anergy. In the last decade, substantial resources have been invested to identify, characterize, and purify various schistosome antigens for the purpose of designing and testing potential vaccines. In fact, elucidation of egg antigens has received much less attention. The importance of pursuing a systematic elucidation of the major egg antigens, resides in the exciting possibility of specifically desensitizing the $\mathrm{CD} 4^{+} \mathrm{Th}$ cells that mediate granuloma formation, which may achieve meaningful prevention or amelioration of clinical disease (Stadecker \& Hernandez 1998). Thus, the present study was carried out to identify Smp40 using molecular modeling, in addition, to characterize the immune response of Thelper cells by estimation of different cytokines patterns after stimulation of peripheral blood mononuclear cells (PBMC) from patients infected with S. mansoni with purified Smp40 major egg antigen.

\section{MATERIALS AND METHODS}

Materials - All chemicals used in this study were of analytical and molecular biology grade and were purchased from Sigma Chemical Co. (St. Louis, MO, US) unless mentioned otherwise and used without further purification.

Expression of S. mansoni Smp 40 protein - The Smp40 gene was cloned and the protein was expressed in pGEX$2 \mathrm{~T}$ vector. The $\mathrm{cDNA}$ containing the entire coding region of Smp40 was cloned into BamHI/EcoR1 site of pGEX-2T. The fusion protein was expressed by the method of Fikrig et al. (1990). Briefly, the glycerol stock culture was thawed at room temperature, then it was inverted several times. A loop of bacteria was streaked into LB plate containing ampicillin $100 \mu \mathrm{g} / \mathrm{ml}$, then incubated at $37^{\circ} \mathrm{C}$ overnight. Single colony was transferred into a $100 \mathrm{ml}$ of LB/amp $(100 \mu \mathrm{g} / \mathrm{ml})$ broth and was incubated overnight at $37^{\circ} \mathrm{C}$ with consistent shaking. Twenty five $\mathrm{ml}$ of the overnight culture was added to 11 of fresh LB/amp media and incubated with shaking at $37^{\circ} \mathrm{C}$ until the $\mathrm{OD}_{600}$ reach between 0.3 to 0.4 . Protein expression was induced by adding 0.25 $\mathrm{mM}$ IPTG. Cells were harvested by spinning for $20 \mathrm{~min}$ at $5000 \mathrm{rpm}$. The pellet was resuspended in $50 \mathrm{ml} 1 \times$ PBS buffer containing $5 \mathrm{mM} \mathrm{MgCl}_{2}$ and $0.2 \mathrm{mM}$ EDTA. Resuspended cells were transferred to sterile tubes and spined at $5000 \mathrm{rpm}$ for $10 \mathrm{~min}$ at $4^{\circ} \mathrm{C}$. The cell pellet was resuspended in $50 \mathrm{ml}$ of $1 \times$ PBS buffer containing $5 \%$ glycerol, then kept at $-80^{\circ} \mathrm{C}$ till use.

Purification of fusion proteins using glutathione column - Reagents used were purchased from Pharmacia Inc., Sweden. Cells that have been previously induced and freezed were thawed at $37^{\circ} \mathrm{C}$. Then, the tubes containing cells were vortexed and sonicated on ice to fully disrupt the cell membrane for releasing the expressed proteins. The suspension was transferred into a glass corex tube and centrifuged at $14,000 \mathrm{rpm}$ for $20 \mathrm{~min}$ at $4^{\circ} \mathrm{C}$. The lysate was saved and filtered through a $0.45 \mu \mathrm{m}$ pore filter. A sample for SDS-PAGE was taken. Dialysis against 2000 $\mathrm{ml} \mathrm{PBS}$ was performed for at least $7 \mathrm{~h}$ at $4^{\circ} \mathrm{C}$. PBS was changed at least every $2 \mathrm{~h}$ (Johnson et al. 1989). The pellet was resuspended in $2 \mathrm{M}$ urea solution for $15 \mathrm{~min}$ at room temperature, sonicated on ice, and centrifuged at $14,000 \mathrm{rpm}$ for $20 \mathrm{~min}$ at $4^{\circ} \mathrm{C}$. After centrifugation, the pellet was discarded and the supernatant was filtered through $0.45 \mu \mathrm{m}$ pore filter and dialyzed against $2000 \mathrm{ml}$ PBS at least $7 \mathrm{~h}$ at $4^{\circ} \mathrm{C}$ with gentle stirring. Changing of PBS buffer was carried out at least every $2 \mathrm{~h}$.

Preparation of glutathione sepharose $4 B$ - The method described by Smith and Johnson (1988) was used. Briefly, glutathione sepharose 4B (GS4B) was resuspended and a sufficient slurry was removed using a pipette and transferred to a tube. The matrix was sedimented by centrifugation at $500 \times \mathrm{g}$ for $5 \mathrm{~min}$ and the supernatnat was decanted. GS4B was washed using $10 \mathrm{ml}$ of cold $1 \times$ PBS buffer per $1.33 \mathrm{ml}$ of the dispensed original slurry of GS4B. The matrix was sedimented by centrifugation at $500 \times \mathrm{g}$ for $5 \mathrm{~min}$ and the supernatant was decanted. A glutathione sepharose column was washed with $10 \mathrm{ml}$ bed volumes of regenerating buffer [high $\mathrm{pH}$ regenerating buffer $(0.5 \mathrm{M}$ $\mathrm{NaCl}$ in $0.1 \mathrm{M}$ Tris- $\mathrm{HCl}, \mathrm{pH} 8.5$ )] followed by the low $\mathrm{pH}$ regenerating buffer $(0.5 \mathrm{M} \mathrm{NaCl}$ in $0.1 \mathrm{M}$ sodium acetate, $\mathrm{pH}$ 4.5). This was repeated three to four times to give a total of four to five washing cycles of alternate buffers. Reequilibration with 3-5 bed volumes of $1 \times$ PBS was carried out.

Batch purification of fusion proteins using bulk glutathione sepharose $4 B$ - Two $\mathrm{ml}$ of glutathione sepharose $4 \mathrm{~B}(50 \%$ slurry) was added and equilibrated with $1 \times$ PBS to each $100 \mathrm{ml}$ of sonicated, filtered, and dialyzed lysate. The lysate-glutathione sepharose 4B preparation was incubated with gentle agitation at room temperature for 30 min. A pipette was used to transfer the matrix to a disposable column. All centrifugations were carried out at $500 \times$ $\mathrm{g}$ for $5 \mathrm{~min}$. The column was tapped to dislodge any trapped air bubbles in the matrix bed and allowed to settle. The column was allowed to drain. The majority of the column flow through was discarded. However, a sample was retained for analysis by SDS-PAGE to measure the efficiency of binding to the matrix. The matrix was washed by the addition of 10 bed volumes of $1 \times$ PBS and the column was drained. The washing was repeated twice more for a total of three washes (Smith \& Johnson 1988).

Elution - The fusion protein was eluted by addition of $1 \mathrm{ml}$ of glutathione elution buffer per $1 \mathrm{ml}$ of glutathione sepharose slurry. The column was incubated at room temperature for $10 \mathrm{~min}$ to elute the fusion protein. The cap was removed and the eluate containing the protein was collected. The elution and collection steps were repeated three times and the eluates were pooled together.

Thrombin cleavage - The method described by Johnson et al. (1989) was followed. Briefly, 10 units of thrombin protease solution was added to $1 \mathrm{mg}$ of fusion protein (eluted from either batch or column chromatography). Then mixed gently and incubated at room temperature for $16 \mathrm{~h}$. Upon completion of enzymatic cleavage, glutathione-S-transferase was removed by extensive dialysis against $1 \times$ PBS followed by batch or column purification on glutathione sepharose. The purified Smp40 was 
recovered in the flow through fraction. A sample was taken for SDS-PAGE analysis for each step of the protocol.

Bicinochoninic acid (BCA) protein assay - The Smp40 concentration was determined using the BCA micromethod protein estimation using BCA kit and protein standards purchased from Pierce Chemicals Co. (Rockford, IL, US). The protocol described by the manufacturer's instructions was applied. The absorbance was measured at $562 \mathrm{~nm}$ on UV microplate reader (Molecular Devices Corporation, Sunnyvale, CA, US).

SDS-PAGE and Western blotting - To analyze samples at different purification steps, sodium dodecyl sulfatepolyacrylamide gel electrophoresis (SDS-PAGE) $0.75-\mathrm{mm}$ thick $12 \%$ vertical slab gels was performed under nonreducing conditions as described by Laemmli (1970). Samples were mixed with an equal volume of sample buffer [0.125 M Trisma base, 4\% (w/v) SDS, 20\% (v/v) glycerol, $10 \%(\mathrm{v} / \mathrm{v})$ mercaptoethanol, $0.1 \%(\mathrm{w} / \mathrm{v})$ bromophenol blue as a tracking dye] and immediately boiled for $5 \mathrm{~min}$. All reagents used were of electrophoresis grade (Bio-Rad Laboratories, Richmond, CA). Mixtures of reference proteins were prepared in parallel (Novex Invitrogen, San Diego, CA). The gels were stained with Commassie brilliant blue $0.05 \%$. The precasted gel (Novex) using their electrophoresis apparatus were used. The separated proteins were transferred electrophoretically onto nitrocellulose paper. The membranes were blocked with $0.3 \%$ PBSTween 20 for $1 \mathrm{~h}$ at room temperature then cut into strips. The strips were incubated with anti-Smp40 monoclonal antibodies and sera (1:100 dilutions) overnight at room temperature with shaking. After washing with PBS-Tween 20 several times (5-6 times), they were incubated in alkaline-phosphatase labeled goat anti-mouse $\mathrm{IgG}$ diluted 1:3000 with $50 \mathrm{mM}$ Tris buffer, $150 \mathrm{mM} \mathrm{NaCl}, \mathrm{pH} 9.6$ for 20 min. After 5-6 times washing with PBS-Tween 20, the strips were exposed to a substrate of $1.65 \mathrm{mg} / \mathrm{ml}$ nitro blue tetrazolium (NBT) and $0.83 \mathrm{mg} / \mathrm{ml}$ 5-bromo-chloro-3-indolyl phosphate (BCIP) (Bio Rad) in $50 \mathrm{mM}$ Tris buffer, $150 \mathrm{mM}$ $\mathrm{NaCl}, \mathrm{pH} 9.6$ supplemented with $5 \mathrm{mM} \mathrm{MgCl}_{2}$. The reaction was stopped with $\mathrm{H}_{2} \mathrm{O}$.

Modeling of Smp40 - Modeling of Smp40 was carried out using software tools for analysis of amino acid and nucleotide sequences alignment. The software includes, BLAST (basic local alignment search tool) (Rastogi 2000); FASTA (Pearson 2000); PILE-UP (Womble 2000); CLUSTAL-W (Rastogi 2000). For Smp40 3D modeling and molecular graphics, these programs were used: 3D-PROFILE (Bowie et al. 1996); TOPITS (Rost 1996); MOLMOL, PHD, MASIA, DIAMOD and FANTOM (Schaumann et al. 1990).

Study population - This study was conducted on 31 $S$. mansoni patients with age range from 19 to 41 years with a mean of 30 years. The patients were selected according to their clinical symptoms (Gazzinelli et al. 1983). The intensity of infection was determined by quantitative egg counts using Kato-Katz procedure. The diagnozed schistosomiasis patients who had other chronic diseases such as diabetes, liver or renal failure and chronic infec- tions were excluded. Fecal samples were collected on three consecutive days to determine the number of $S$. mansoni eggs. A blood sample withdrawn from each patient, anticoagulated with heparin and subjected to density gradient centrifugation at $1500 \mathrm{rpm}$ at $22^{\circ} \mathrm{C}$ for $40 \mathrm{~min}$. PBMC were prepared according to the method mentioned by Gazzinelli et al. (1983). The study protocol respected the most recent Declaration of Helsinki (Edinburgh 2000). All of the patients gave consent to the use of their blood and clinical data for research purposes after being informed about the nature of the study.

SEA preparation - SEA was isolated from crude homogenates of $S$. mansoni eggs (Doughty \& Phillips 1982). Parasite eggs were isolated from livers of 7 to 8 week infected outbred ICR mice. The livers were homogenized extensively using a waring blender in $1.7 \% \mathrm{NaCl}$ in $0.15 \mathrm{M}$ phosphate buffered saline, $\mathrm{pH} 7.4(2 \times \mathrm{PBS})$ followed by differential sieving, repeated sedimentation, and low-speed centrifugation to remove all tissue proteins and to separate the eggs from contaminating liver tissue. Eggs were further purified by enzymatic digestion with pronase, followed by DNase digestion to remove all tissue debris. Additional washes with $2 \times$ PBS were performed until the parasite eggs were clean upon microscopic observation. Fully embryonated eggs were separated from dead or immature eggs on a stepwise Percoll gradient. Eggs were washed again in $2 \times$ PBS, counted and resuspended to give a suspension of 50,000 eggs $/ \mathrm{ml}$ in sterile PBS. This suspension was homogenized in a tissue homogenizer with a motor driven Teflon pestle. The homogenate was centrifuged at $100,000 \times \mathrm{g}$ at $4^{\circ} \mathrm{C}$ for $2 \mathrm{~h}$. The supernatant was collected, sterilized through a $0.45 \mathrm{~mm}$ filter and stored frozen at $-70^{\circ} \mathrm{C}$ for later use as SEA. SEA protein content was determined using the Bicinochoninic acid (BCA) protein assay according to manufacturer's instructions (Pierce Chemical Co.).

Cytokines determination by ELISA - PBMC were isolated from two volumes of heparinized blood of patients infected with S. mansoni using one volume of Ficoll-sodium diatrizoate by density gradient centrifugation at 400 $\times \mathrm{g}$ for $45 \mathrm{~min}$ at $25^{\circ} \mathrm{C}$ (Gazzinelli et al. 1983). The resulting PBMC layer was washed three times in incomplete RPMI 1640, and resuspended in complete RPMI 1640 supplemented with $10 \%$ human $\mathrm{AB}^{+}$serum (RPMI-HS) (Gemini Products, Calabasa, CA), $20 \mathrm{mM}$ L-glutamine, 3\% antibiotic/ antimycotic (containing penicillin, streptomycin, fungizone, and gentamicin), a MEM non- essential amino acid solution $0.1 \mathrm{mM}$, and sodium pyruvate $1.0 \mathrm{mM}$. Cell count was carried out using a hemocytometer. PBMC $(3 \times$ $10^{6} \mathrm{PBMC} / \mathrm{ml}$ ) were stimulated with phytohemagglutinin (PHA) mitogen as a positive control in a final dilution of 1:100 (Gibco), SEA and Smp40 antigens for 24, 48, and 72 h. Negative control PBMC was incubated as above without stimulation with neither mitogens nor antigens. The cytoscreen ELISA kits were purchased from Biosource International (California, US). Levels of IFN- $\gamma$, IL-4, IL-5, IL-10, and IL-13 were measured in supernatants of stimulated PBMC. The levels of cytokines were determined by using a sandwich ELISA technique. A standard curve was used to express the results in picograms per milliliter. The 
microplate measured at $450 \mathrm{~nm}$ on UV microplate reader (Molecular Devices Corporation, Sunnyvale, CA) within 30 min of adding stop solution for all standards, controls, and samples. ELISA assay method and preparation of standards or reagents were performed as described by manufacturer's instructions.

Statistical analysis and data presentation - Figures show data compiled from several experiments, or from a representative experiment, as specified. Results represent the mean $\pm \mathrm{SD}$, where applicable. Statistical significance of differences was analyzed using Mann-Whitney test, or Kruskal-Wallis test as appropriate. The Mann-Whitney test was used to compare between two groups for continuous variable. Kruskal-Wallis test was performed to compare three or more different groups. Values of $P \leq 0.05$ were considered significant. All statistical procedures were performed by using SPSS software, version 11 for windows (SPSS Inc., US).

\section{RESULTS}

Purification of Smp40 - GST fusion protein was purified using the affinity matrix glutathione sepharose $4 \mathrm{~B}$. Commassie blue stained 12\% SDS-PAGE electrophoresis (Fig. 1) shows the purification steps of Smp40. The final purification step of Smp40 was performed by adding the glutathione elution buffer and collecting the flow through solution from the GST column four times to further purify the Smp40 from the other protein contaminants. The elution gave us only two bands the specific protein band (Smp40) and another band was the GST band. Rerunning the eluate on the GST affinity chromatography column for one more time allowed the separation of the Smp40 protein band only, which means complete purification of that protein. All these steps are explained in Figs 1, 2.

Western blot for Smp40 - Western blotting for the Smp40 protein preparation using anti-Smp40 monoclonal antibody showed a specific reaction at molecular weight of $40 \mathrm{KDa}$ (Fig. 3).

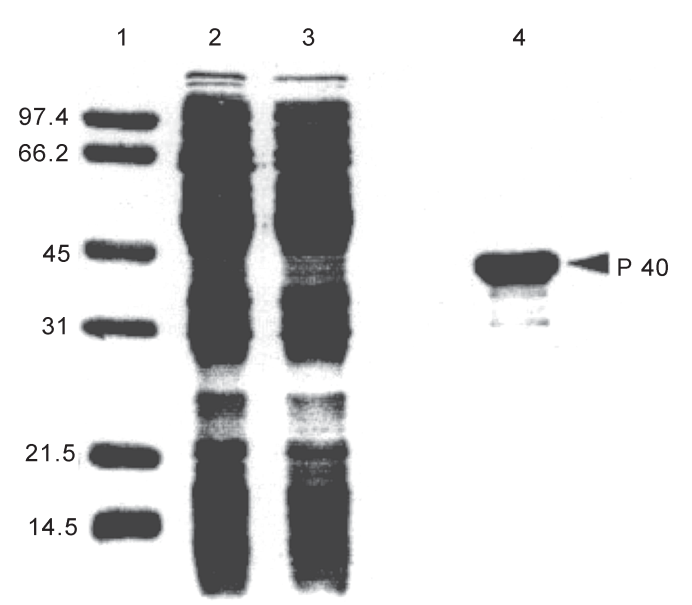

Fig. 1: Coomassie blue stained $12 \%$ sodium dodecyl sulfate-polyacrylamide gel electrophoresis. Lanes - 1: molecular weight markers; 2: Escherichia coli lysate; 3: glutathione chromatography flowthrough fraction; 4: partially purified fraction after thrombin cleavage.

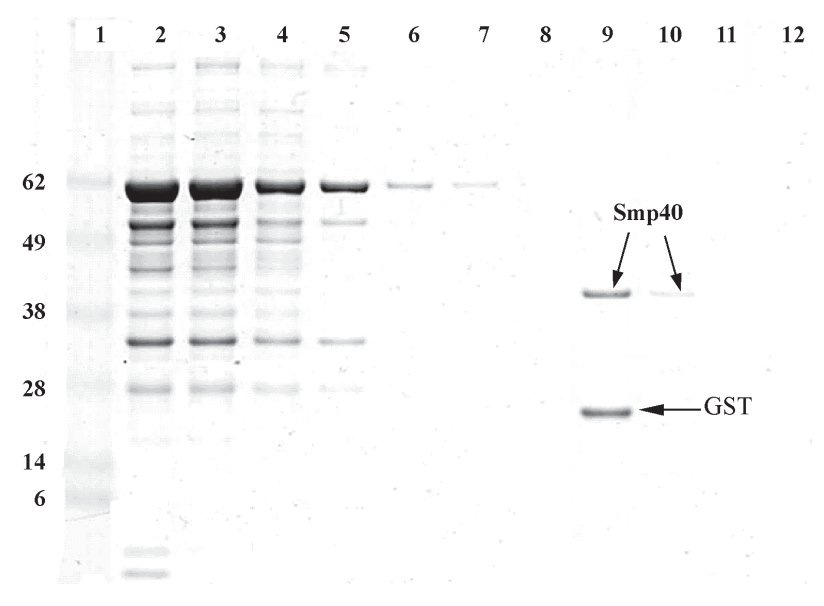

Fig. 2: the final purification steps of Smp40 using glutathione-Stransferase affinity chromatography, elution, and cleavage by thrombin protease. $12 \%$ sodium dodecyl sulfate-polyacrylamide gel from Novex Invetrogin Corporation. Lanes - 1: see blue prestained molecular weight marker; 2: lysate (sonicated, centrifuged, and filter sterilized expressed protein); 3: dialyzed supernatants (lysate after centrifugation and dialyzed against $1 \times$ phosphate-buffer saline (PBS); 4: flow through glutathione sepharose column; 5: eluate (4 times repeat); 6: wash $1(1 \times \mathrm{PBS})$ after elution; 7: wash $2(1 \times$ PBS $)$ after elution; 8: wash $3(1 \times$ PBS $)$ after elution; 9: eluate dialyzed against $1 \times \mathrm{PBS}$, the eluate contains two bands, the upper one is the Smp40 protein and the lower one is the glutathione-S-transferase (GST) band; lane 10: Smp40 after thrombin cleavage, shows only the Smp40 band after cleavage with thrombin which revealed the Smp40 from the GST beads and set it free in the flow through; 11: wash $1(1 \times$ PBS $)$; 12 : wash $2(1 \times$ PBS $)$.

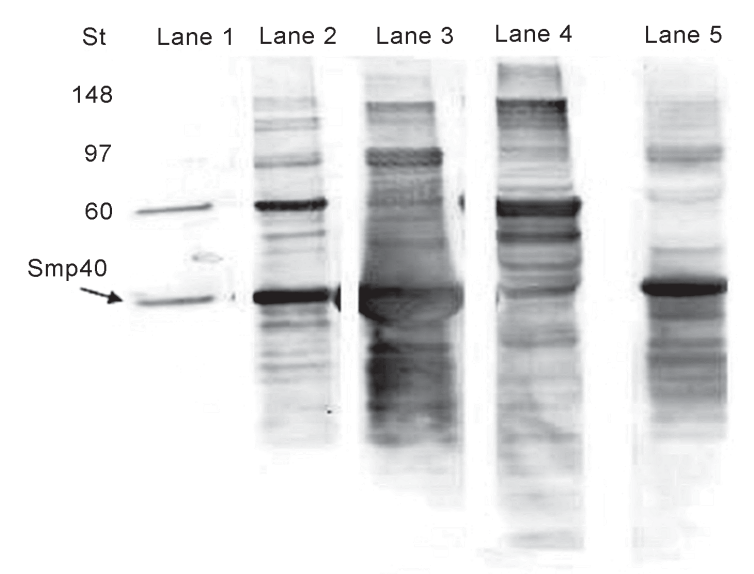

Fig. 3: Western blot of Smp40 preparations with anti-Smp40 monoclonal antibody. Lanes - 1: Smp40; 2: Smp40 before second glutathione sepharose column; 3: flow through fraction; 4: Smp40 lysate; 5: soluble egg antigens.

Smp40 modeling - Three-dimensional structure was elucidated based on the similarity of Smp40 with the small heat shock protein (HSP) coded in the protein database (PDB) as 1SHS as a template in the molecular modeling. It was figured out that the C-terminal of the Smp40 protein (residues 130 onward) contains two alpha crystallin domains. The fold consists of eight beta strands sandwiched in two sheets forming Greek key. The model was generated using EXDIS-DIAMOD-FANTOM programs (Figs 4,5). 
P $40 / 75-129$
SHS / 1-44
P $40 / 129-224$
1 SHS / 4 5end

P40/242end 1 SHS / 4 5end

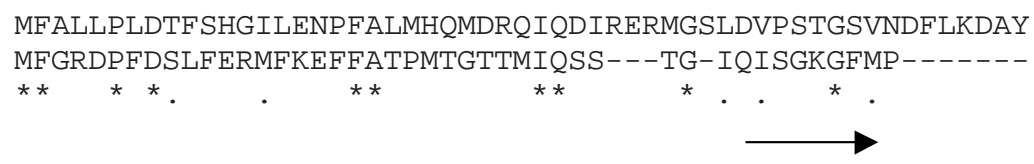

YEVGEDGKVHFKVRFDAQGFAPQD INVTSSENRVTVHAKKETTTDGRKCSREFCRMVQLPK IS I IE-GDQH I KVIAWLP GVNKED I I LNAVGDTLEIRAKRSP------------LMITESE

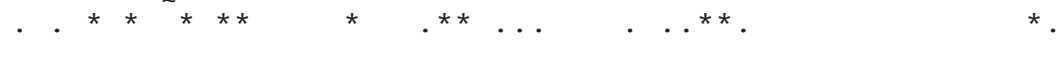

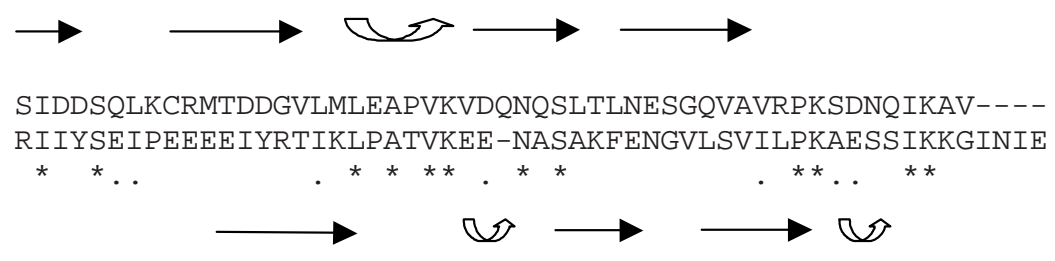

PASQALVAKGVHGLSYVDDGSGGKRLHVEVPVDPVYNPEDLCVNVDSNRVVVSGRHHKQK ----------- IS I IE---G-DQH I KVIAWLP GVNKED I I LNAVGDTLEIRAKRSP LM

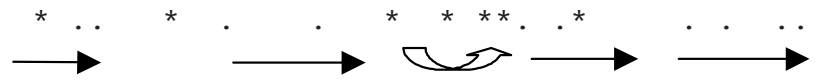

SDQ-----HGRSS SFAEF SQSYAIPETVDPLSVSAQVVGNTLVLEAPLEKQHAITH---ITESERI IYSEIPEEEEIYRTIKLPATVKEENASAKFENGVLSVILPKAESS IKKGINIE

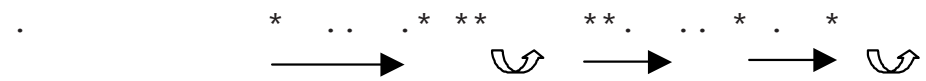

Fig. 4: suggested alignment and corresponding secondary structure for residues 75 -end of P40 and the small heat shock protein 20 of Methanococcus janschenii (protein database file 1SHS). Straight arrows indicate beta strands, curved, 3-10 helices, in the SHS structure.

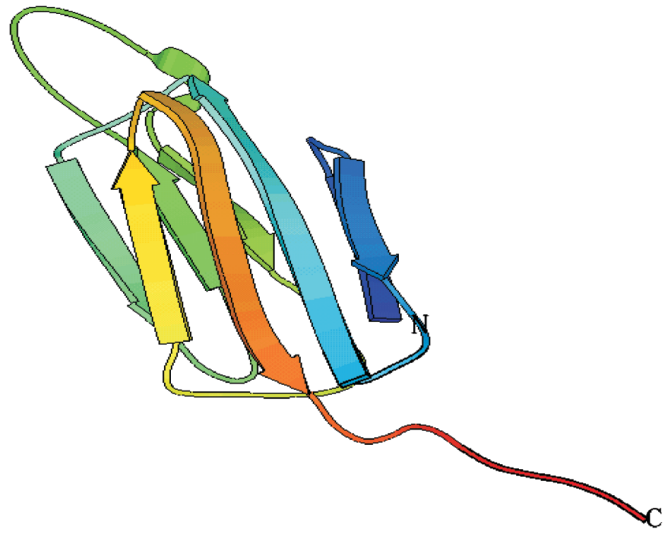

Fig. 5: model of the Smp40 residues 121-228 using 1 SHS as a template. The fold consists of eight beta strands sandwiched in two sheets forming Greek key. The model was generated using EXDISDIAMOD-FANTOM programs.

Cytokines level - The generated and purified protein was used for in vitro stimulation of PBMC of patients infected with $S$. mansoni; these patients were chosen in the young age group, almost half males and half females to exclude the effect of the age or sex on the immune response to infection. The younger patients have more vigorous $T$ cell responsiveness to SEA and SEA-HPLC fractions than the older patients suggesting that SEA responses wane as the infection progresses.

The purified Smp40 was used for in vitro stimulation of PBMC from patients infected with $S$. mansoni along with total SEA using PHA mitogen as a positive control.
Consistent results were obtained with $72 \mathrm{~h}$ incubation. The results showed that there is no statistical difference between the IFN- $\gamma$ level obtained with Smp40 and SEA stimulation compared with the control group $(P>0.05)$. No statistical difference was found in IL-4 level after stimulation with Smp40, SEA, and PHA compared with the control $(P>0.05)$. Concerning IL-5, there was a statistical insignificance between PHA and SEA $(P>0.05$ for each) compared with the control, but in case of Smp40, there was a significant difference with the control $(P=0.006)$. IL-10 increased significantly with stimulation with PHA, SEA and Smp40 compared with the control group $(P<$ $0.001,0.002, P<0.001$ respectively). On the other hand, in comparison with control group, IL-13 was statistically different in case of PHA and SEA stimulation $(P<0.001$ for each) and insignificant in case of Smp40 $(P>0.05)$ (Table).

\section{DISCUSSION}

Gaining knowledge about genomes of schistosome parasites has been increasingly important for the understanding of parasite biology, drug resistance mechanisms, and antigenic variation that determine escape from the host's immune system. Every attempt is made towards controlling the dispersal of parasitic diseases in developing countries, either by discovering novel therapeutic drugs, new diagnosis tests or by producing effective vaccines.

The Smp40 antigen has been independently described in several laboratories (Nene et al. 1986, Cao et al. 1993), and has been sequenced and cloned. The full nucleotide and deduced 354 amino acid sequence of the Smp40 antigen have been published by Nene et al. (1986). The se- 
TABLE

Cytokines pattern measured in extracted supernatants of cultured peripheral mononuclear cells of patients with Schistosoma mansoni after $72 \mathrm{~h}$ of stimulation with soluble egg antigens and Smp40 antigens in comparison with phytohemagglutinin mitogen

\begin{tabular}{lccccc}
\hline Cytokine $(\mathrm{pg} / \mathrm{ml})$ & Control & PHA & SEA & Smp40 & $P$ Value \\
\hline IFN- $\gamma$ & $24.64 \pm 6.6$ & $903.4 \pm 264.5$ & $27.6 \pm 3.2$ & $26.1 \pm 4.6$ & $P>0.05$ \\
IL-4 & $41.93 \pm 16.3$ & $17.3 \pm 2.5$ & $13.4 \pm 2.1$ & $7.9 \pm 1.4$ & $P>0.05$ \\
IL-5 & $103.4 \pm 16.4$ & $94.2 \pm 13.1$ & $99.59 \pm 17.5$ & $56 \pm 23.5$ & $P=0.006$ \\
IL-10 & $25.8 \pm 3.9$ & $410.3 \pm 61$ & $59.4 \pm 11.3$ & $302.8 \pm 67.5$ & $P<0.001$ \\
IL-13 & $8 \pm 1.2$ & $246 \pm 53.7$ & $132.6 \pm 39.7$ & $5.5 \pm 1.2$ & $P>0.05$ \\
\hline
\end{tabular}

IFN- $\gamma$ : interferon-gamma; IL: interleukin; data are expressed as mean \pm SE; $P$ value obtained as a result of comparison of Smp40 to the control group.

quence of Smp40 was carried out and published elsewhere by Prof. Doughty's team (Cao et al. 1993). It was different from the sequence determined by Nene et al. (1986) in some amino acids.

The fold of Smp40 was identified using threading techniques. Almost all the threading methods predicted with significant score that the core of the residues 121-228 of Smp40 sequence have similar fold like that of small heat shock protein (PDB code-1SHS) from Methanococcus jannaschii (Kim et al. 1998). 1SHS belongs to the protein class of all beta protein and has a fold characterized by sandwich of eight beta strands in two sheets forming a Greek-key, 106 residues out of 360 residues were modeled. Distance and angle constraints were obtained from the aligned region with the template. Flexibility for the target was allowed by setting a threshold of 0.5 for the upper and lower limits of the constraints. A total of 12,780 were extracted. Unsuitable constraints were removed by running the DIAMOD program (Schaumann et al. 1990) and to generate an initial structure. The crude structure was subjected to energy minimization using FANTOM (fast Newton-Raphson torsion angle minimizer) program (Guntert et al. 1991). The final structure was obtained without restraints. PROCHECK analysis (Laskowski et al. 1993) showed that $99 \%$ of residues are in the allowed region. The model has eight beta-strands sandwiched in two sheets forming Greek key. There is large loop made of residues 51-62.

In schistosomiasis mansoni, the chronic egg-induced granulomatous response in the liver and intestine may eventually cause extensive tissue scarring and development of portal hypertension. Indeed, much of the morbidity and mortality associated with this disease is directly attributable to the deposition of connective tissue elements in affected tissues. Elucidating the mechanisms that regulate the severity of schistosomiasis has been a major research objective over the past several years.

IFN- $\gamma$ plays an immunoregulatory role at different stages of the experimental $S$. mansoni-driven processes (Asseman et al. 1996). The present study showed that IFN- $\gamma$ increased significantly with PHA and insignificantly with SEA and Smp40. There is a recent study that correlates the enhanced egg-induced immunopathology with high IFN- $\gamma$ in murine schistosomiasis (Rutitzky et al. 2005). Mwatha et al. (1998) reported that the higher IFN- $\gamma$ than the controls is associated with hepatosplenic disease in human S. mansoni. In murine schistosomiasis, Hernandez et al. (1998) reported that the cells responding to Smp40 antigen secreted high level of IFN- $\gamma$. These different findings in the different model introduce the important notion that egg antigens can vary significantly in immunogenicity according to the host's genetic background (Stadecker et al. 2001).

In the pulmonary granuloma model, IFN- $\gamma$ inhibits granuloma formation; however, the role of IFN- $\gamma$ in hepatic granulomatous inflammation remains unclear. There is considerable evidence that IFN- $\gamma$ participates in development of hepatic granuloma. IFN- $\gamma$ is produced by hepatic granuloma at the time of peak inflammation, and, in some studies, animals deficient in IFN- $\gamma$ or IFN- $\gamma$ receptor or treated with anti-IFN- $\gamma$ antibody had an impaired hepatic granulomatous inflammation. Other investigators who used similar types of IFN- $\gamma$ or IFN- $\gamma$ receptor-deficient mice failed to observe any effect on $S$. mansoni hepatic granulomatous reactions. Local recruitment of egg antigen-specific lymphocytes and eosinophils to sites of egg deposition may exacerbate granulomatous inflammation through enhanced local production of cytokines such as IL-5 (reviewed in King et al. 2001).

The initial immune response to $S$. mansoni eggs presumably results in IL-4 production, as schistosome eggs are strong Th2 inducing antigens and the differentiation of antigen-specific Th2 cells is largely dependent on the presence of IL-4 during priming of naïve Th cells (Sabin et al. 1996). The present results showed that IL-4 decreased after stimulation with PHA, SEA, and Smp40 in comparison with the control group. The difference was statistically insignificant. The low level of IL-4 is associated with reduced inflammation, markedly decreasing the hepatic collagen deposition and reduces fibrosis (Cheever et al. 1994). In accordance with our results, Hernandez et al. (1998) reported that the cells responding to Smp40 antigen showed a non-detectable level of IL-4.

The most interesting findings obtained with the cytokines results after stimulation of PMBC with Smp40 are: (1) the major induction of IL-10; (2) the low level of IL13 detected upon stimulation with Smp40 that associated with low level of IL-5. IL-10 is able to inhibit the production of pro-inflammatory mediators such as IFN- $\gamma$, TNF$\alpha$, and NO (Royer et al. 2001). IL-10 reduces the granuloma formation when administered in vivo (FloresVillanueva et al. 1996). Exogenous IL-10 can reverse CCl4induced hepatic fibrosis in rats. IL-10 may exert its reversible effects on hepatic fibrosis by blocking CCl4-induced inflammation, inhibiting expression of matrix metalloproteinase-2 (MMP-2) and tissue inhibitor of matrix 
metalloproteinase-1 (TIMP-1) and promoting resolution of collagen types I and III (Huang et al. 2006). Worth noting, S. mansoni infections, through induction of regulatory mechanisms, such as IL-10 production, are able to modulate positively the inflammatory immune response involved in the pathology of autoimmune and allergic disease (Araujo et al. 2004). Surprisingly, a strong association between egg antigen-driven TNF- $\alpha$ production and diminished IL-10 release and bladder pathology in $S$. haematobium-infected children and adolescents. The increased production of IL-10, a cytokine that defies clear Th1/Th2 categorization and is associated with immune modulation in urinary schistosomiasis, may help to prevent bladder disease (King et al. 2001).

IL-10 deficient mice spontaneously develop inflammatory bowel disease, which is most likely due to dysregulated production of cytokines. In addition, IL-10 has been shown to be crucial for protection of mice from acute lipopolysaccharide-induced endotoxic shock. Thus, it becomes increasingly clear that IL-10 has a role in the down-regulation of cell-mediated immune hyperreactivity. IL-10 deficient mice infected with Toxoplasma gondii die early in infection, show elevated levels of serum IL-12 and IFN- $\gamma$. In addition, the macrophages from IL-10 deficient mice produced higher levels of TNF- $\alpha$ and IL-12 after stimulation with $T$. gondii compared to macrophages from experimental mice. It was found that IL-10 deficient mice succumb to infection with $T$. gondii due to high levels of inflammatory cytokines IL- 12 and IFN- $\gamma$ (Neyer et al. 1997).

The IL-5 is a hormone of the immune system that is the main regulator of eosinopoiesis, eosinophil maturation and activation, and immunoglobulin A production. The results showed that IL-5 decreased with Smp40 significantly $(P<$ $0.05)$. In SCID mice, the reduction of egg-associated pathology was associated with low level of IL-5 (Cheever et al. 1999). Blocking of IL-13 in experimental animals almost completely abrogated granuloma development and pulmonary eosinophilia (Chiaramonte et al. 1999). The present results showed that the levels of IL-13 was increased significantly with PHA and SEA $(P<0.05)$, but was at the same level of the control group after stimulation with Smp40. The low level of IL-13 is associated with reduced inflammation, markedly decreasing the hepatic collagen deposition and reduce fibrosis (Cheever et al. 1994). Recently, it was found that IL-5 augments the progression of liver fibrosis by regulating IL-13 activity. They found that granuloma eosinophils were themselves a significant source of IL-13 and concluded that there are both direct and indirect roles for eosinophils and IL-5 in the pathogenesis of schistosomiasis-induced liver fibrosis. Thus, inhibiting the activity of IL-5 or eosinophils may prove effective for a variety of chronic fibrotic diseases (Reiman et al. 2006). These results are in accordance with those obtained by Alves Oliveira et al. (2006) who reported that IL-13 was strongly associated with fibrosis (odds ratio = 5.8 ) in schistosomiasis mansoni in humans. de Jesus et al. (2004) observed significant increases in IL-5 and IL-13 levels in some of the subjects with fibrosis who remained untreated for one year following initial assessment and developed more serious fibrosis during this period.

The Th2-like response to ova antigens in humans may be the normal anti-inflammatory, antipathologic response to prevent hepatic fibrosis and intestinal inflammation. Therefore, from the cytokine profile obtained by Smp40 stimulation, it seems clear that Smp40 may be one of the most promising antigens as anti-pathology leading vaccine candidate for this parasite, for its exciting possibility for reducing collagen deposition, decreasing fibrosis and inhibiting granuloma formation.

\section{ACKNOWLEDGMENT}

To CH Schein, Human Biological Chemistry and Genetics Department, The University of Texas Medical Branch at Galveston, for assistance during this work and for the distinguished professors SM Phillips, PT LoVerde, and DA Harn for kindly supplying the Smp40 cDNA and anti-Smp40 monoclonal antibody.

\section{REFERENCES}

Asseman C, Pancer V, Quatennens B, Auriault C 1996. Schistosoma mansoni infected mice show augmented hepatic fibrosis and selective inhibition of liver cytokine production after treatment with anti-NK-1.1 antibodies. Immunol Lett 54: 11-20.

Alves Oliveira LF, Moreno EC, Gazzinelli G, Martins-Filho OA, Silveira AM, Gazzinelli A, Malaquias LC, LoVerde P, Leite PM, Correa-Oliveira R 2006. Cytokine production associated with periportal fibrosis during chronic schistosomiasis mansoni in humans. Infect Immun 74: 1215-1221.

Araujo MI, Hoppe BS, Medeiros Jr M, Carvalho EM 2004. Schistosoma mansoni infection modulates the immune response against allergic and autoimmune diseases. Mem Inst Oswaldo Cruz, 99 (Suppl. 1): 27-32.

Bahia-Oliveira LM, Gazzinelli G, Eloi-Santos SM, Cunha-Melo JR, Alves-Oliveira LF, Silveira, AMS, Viana IRC, Carmo J, Souza A, Correia-Oliveira R 1997. Differential cellular reactivity to adult worm antigens of patients with clinical forms of Schistosomiasis mansoni. Tran R Soc Trop Med Hyg 86: 57-61.

Bowie JU, Zhang K, Wilmanns M, Eisenberg D 1996. Threedimensional profiles for measuring compatibility of amino acid sequence with three-dimensional structure. Meth Enzymol 266: 598-616.

Cao M, Chao H, Doughty BL 1993. Cloning of a cDNA encoding an egg antigen homologue from Schistosoma mansoni. Mol Biochem Parasitol 58: 169-171.

Cheever AW, Williams ME, Wynn TA, Finkelman FD, Seder RA, Cox TM, Hieny S, Caspar P, Sher A 1994. Anti-IL-4 treatment of Schistosoma mansoni-infected mice inhibits development of T cells and non-B, non-T cells expressing Th2 cytokines while decreasing egg-induced hepatic fibrosis. J Immunol 153: 753-759.

Cheever AW, Poindexter RW, Wynn TA 1999. Egg laying is delayed but worm fecundity is normal in SCID mice infected with Schistosoma japonicum and S. mansoni with or without recombinant tumor necrosis factor alpha treatment. Infect Immun 67: 2201-2208.

Chiaramonte MG, Donaldson DD, Cheever AW, Wynn TA 1999. An IL-13 inhibitor blocks the development of hepatic fibrosis during a T-helper typ2-dominated inflammatory response. J Clin Invest 104: 777-785.

de Jesus AR, Magalhães A, Miranda DG, Miranda RG, Araujo MI, de Jesus AA, Silva A, Santana LB, Pearce E, Carvalho 
EM 2004. Association of type 2 cytokines with hepatic fibrosis in human Schistosoma mansoni infection. Infect Immun 72: 3391-3397.

Doughty BL, Phillips SM 1982. Delayed hypersensitivity granuloma formation around Schistosoma mansoni eggs in vitro. I. Definition of the model. J Immunol 128: 30-36.

El-Zayadi AR 2004.Curse of schistosomiasis on Egyptian liver. World J Gastroenterol 10: 1079-1081.

Flores-Villanueva PO, Zheng XX, Strom TB, Stadecker MJ 1996. Recombinant IL-10 and IL-10/Fc treatment downregulate egg antigen-specific delayed hypersensitivity reactions and egg granuloma formation in schistosomiasis. $J$ Immunol 156: 3315-3320.

Fikrig E, Barthold SW, Kantor FS, Flavell RA 1990. Protection of mice against the Lyme disease agent by immunizing with recombinant OSPA. Science 250: 553-556.

Gazzinelli G, Katz N, Rocha RS, Colley DG 1983. Immune responses during human schistosomiasis mansoni. X. Production and standardization of an antigen-induced mitogenic activity by peripheral blood mononuclear cells from treated, but not active cases of schistosomiasis. J Immunol 130: 2891-2895.

Goes AM, Hirsch C 1996. Characterization of fractionated $S$. mansoni soluble adult worm antigens that elicit human cell proliferation and granuloma formation in vitro. Parsitology 112: 529-535.

Guntert P, Braun W, Wuthrich K 1991. Efficient computation of three-dimensional protein structures in solution from nuclear magnetic resonance data using the program DIANA and the supporting programs CALIBA, HABAS and GLOMSA. J Mol Biol 217: 517-530.

Hernandez HJ, Edson CM, Harn DA, Ianelli CJ, Stadecker MJ 1998. Schistosoma mansoni: Genetic restriction and cytokine profile of the $\mathrm{CD} 4^{+} \mathrm{T}$ helper cell response to dominant epitope peptide of major egg antigen Sm-p40. Exp Parasitol 90: 122-130.

Huang Y-H, Shi M-N, Zheng W-D, Zhang L-J, Chen Z-X, Wang $\mathrm{X}-\mathrm{Z}$ 2006. Therapeutic effect of interleukin-10 on CCl4induced hepatic fibrosis in rats. World J Gastroenterol 12: 1386-1391

Iacomini J, Ricklan DE, Stadecker MJ 1995. T cells expressing the $\gamma \delta$ T cell receptor are not required for egg granuloma formation in schistosomiasis. Eur J Immunol 25: 884.

Johnson KS, Wells K, Bock JV, Nene V, Taylor DW, Cordingley JS 1989. The 86-kilodalton antigen from Schistosoma mansoni is a heat-shock protein homologous to yeast HSP90. Mol Biochem Parasitol 36: 19-28.

Kim KK, Kim R, Kim SH 1998. Crystal structure of a small heat-shock protein. Nature 394: 595-599.

King CL, Malhotra I, Mungai P, Wamachi A, Kioko J, Muchiri E, Ouma JH 2001. Schistosoma haematobium-induced urinary tract morbidity correlates with increased tumor necrosis factor- $\alpha$ and diminished interleukin-10 production. $J$ Infec Dis 184: 1176-1182.

Laemmli UK 1970. Cleavage of structural proteins during the assembly of the head of bacteriophage T4. Nature 227: 678-685.

Laskowski RA, McArthur MW, Moss DS, Thornton JM 1993. PROCHECK: a program to check the stereochemical quality of protein structures. J Appl Cryst 26: 283-291.
Mwatha JK, Kimani G, Kamau T, Mbugua GG, Ouma JH, Muma J, Fulforf JC, Jones FM, Butterworth AE, Roberts MB, Dunne DW 1998. High level of TNF soluble TNF receptors soluble ICAM-1 and IFN- $\gamma$ but low level of IL-5 are associated with hepatosplenic disease in human $S$. mansoni. J Immunol 160: 1992-1998.

Nene V, Dunne DW, Johnson KS, Taylor DW, Cordingly JS 1986. Sequence and expression of a major egg antigen from Schistosoma mansoni: homologies to heat shock proteins and alpha-crystallins. Mol Biochem Parasitol 21: 179-188.

Neyer LE, Grünig G, Fort M, Remington JS, Rennick D, Hunter CA 1997. Role of interleukin-10 in regulation of T-celldependent and T-cell-independent mechanisms of resistance to Toxoplasma gondii. Infection Immunity 65: 1675-1682.

Pearson WR 2000. Flexible sequence similarity searching with the FASTA3 program package. In Misener S, Krawetz SA (eds), Bioinformatics methods and protocols. Meth $\mathrm{Mol}$ Biol 132: 185-219.

Rastogi PA 2000. MacVector integrated sequence analysis for the Macintosh. In Misener S, Krawetz SA (eds), Bioinformatics methods and protocols. Meth Mol Biol 132: 47-69.

Reiman RM, Thompson RW, Feng CG, Hari D, Knight R, Cheever AW, Rosenberg HF, Wynn TA 2006. Interleukin-5 (IL-5) augments the progression of liver fibrosis by regulating IL-13 activity. Infect Immun 74: 1471-1479.

Rost B 1996. PHD: Predicting one-dimensional protein structure by profile-based neural networks. Meth Enzymol 266: 525-539.

Royer B, Varadaradjalou S, Saas P, Guillosson JJ, Kantelip JP, Arock M 2001. Inhibition of IgE-induced activation of human mast cells by IL-10. Clin Exp Allergy 31: 694-704.

Rutitzky LI, Hernandez HJ, Yim YS, Ricklan DE, Finger E, Mohan C, Peter I, Wakeland EK, Stadecker MJ 2005. Enhanced egg-induced immunopathology correlates with high IFN-gamma in murine schistosomiasis: identification of two epistatic genetic intervals. Immunology 174: 435-440.

Sabin EA, Kopf MA, Pearce EJ 1996. Schistosoma mansoni egg-induced early IL-4 production is dependent upon IL-5 and eosinophils. J Exp Med 184: 1871-1878.

Schaumann TH, Braun W, Wüthrich K 1990. The program FANTOM for energy refinement of polypeptides and proteins using a Newton-Raphson Minimizer in torsion angle space. Biopolymers 29: 679-694.

Smith DB, Johnson KS 1988. Single-step purification of polypeptides expressed in $E$. coli as fusions with glutathione-S-transferase. Gene 67: 31-40.

Stadecker MJ, Hernandez HJ 1998. The immune response and immunopathology in infection with Schistosoma mansoni: a key role of major egg antigen Sm-p40. Parasite Immunol 20: 217-221.

Stadecker MJ, Hernandez HJ, Asahi H 2001. The identification and characterization of new immunogenic egg components: implications for evaluation and control of the immunopathogenic $\mathrm{T}$ cell response in schistosomiasis. Mem Inst Oswaldo Cruz 96: S29-33.

Womble DD 2000. GCG: the Wisconsin package of sequence analysis programs. In Misener S, Krawetz SA (eds), Bioinformatics methods and protocols. Meth Mol Biol 132: 3-22. 